\title{
GOBIERNO CORPORATIVO Y RIESGO CREDITICIO EN EL SISTEMA FINANCIERO PERUANO. UNA PRIMERA APROXIMACIÓN
}

\author{
Portalanza Chinguel, César ${ }^{A}$ | Castro Muro, Fernando | Sotelo Tapia, Nidia
}

\section{RESUMEN}

La gestión del riesgo es la actividad central en la banca, y la gestión del riesgo crediticio es vital para la sostenibilidad del negocio bancario; en ese sentido, buenas prácticas de gobierno corporativo son generalmente asociadas con una mejor gestión del riesgo crediticio. Mediante análisis de correlaciones, usando información pública de instituciones financieras, se han identificado algunas variables de gobierno corporativo y se las asoció a indicadores de gestión de la cartera de créditos. De nuestro análisis, no existe una clara correlación entre gobierno corporativo y gestión del riesgo crediticio; sin embargo, se ha observado una fuerte relación entre tamaño del directorio e indicadores de gestión de la cartera de créditos.

JEL: G21

PALABRA CLAVE: Gobierno corporativo, gestión del riesgo, riesgo de crédito

\begin{abstract}
Risk management is the key activity in banking, and the management of credit risk is vital for the sustainability of the banking business; in that sense, good corporate governance is generally associated with better performance in credit risk management. Through correlation analysis, using public information on financial institutions, some corporate governance variables have been identified and associated with credit portfolio management's performance indicators. As per our observation, there is no clear correlation between corporate governance and credit risk management performance; however, a strong relation between size of the board and credit portfolio management performance has been observed.
\end{abstract}

JEL: G21

KEY WORD: Corporate governance, risk management, credit risk

A César Portalanza es Investigador Docente de la Escuela Profesional de Economía. Fernando Castro y Nidia Sotelo son Economistas de la Escuela Profesional de Economía Facultad de Ciencias Contables, Económicas y Financieras de la Universidad San Martín de Porres (USMP). Lo expresado en el presente artículo es de mi entera responsabilidad y sólo refleja mi opinión personal y no involucra la opinión de las instituciones en las cuales trabajo. Fernando Castro y Nidia Sotelo son economistas de la Escuela Profesional de Economía de la USMP. 


\section{INTRODUCCIÓN}

Prácticas adecuadas de gobierno corporativo permiten fortalecer la mitigación de riesgos, y propicia un fortalecimiento de los mecanismos de control y equilibrio de poderes en las empresas del sistema financiero. Di Benedetta y otros (2015) señalan que el buen gobierno de las instituciones financieras juega un papel crucial en el desarrollo de productos, en el avance tecnológico y más importante aún, en la gestión de crisis ${ }^{1}$.

En ese sentido, se busca una mejora del gobierno corporativo como un mecanismo importante para que las empresas del sistema financiero mejoren su gestión de riesgos y sean sostenibles en el largo plazo $^{2}$ y donde el Directorio asume un rol protagónico, como lo señala Stulz (2016):

...the board has to ensure that the firm has the capability to measure and manage risk so that it has the right level of risk given its risk appetite, and has to ensure that it uses this capability effectively so that it actually takes the right level of risk. This means that the bank should have a risk management organization in place capable of making sure that it has the right level of risk.

Existe un consenso creciente a nivel internacional de que un buen marco de gobierno corporativo beneficia a las empresas. Al respecto, Claessens y Yurtoglu (2012) $)^{3}$ muestran que un buen marco de gobierno corporativo es positivo para las empresas a través de un mayor acceso a financiamiento, menores costos de capital, mejor desempeño financiero y un tratamiento más favorable por parte de los grupos de interés (stakeholders).

Entre los aspectos pendientes que Claessens y Yurtoglu destacan está el poco desarrollo de los temas de gobierno corporativo de las instituciones financieras, especialmente en el caso de los países en desarrollo. Éste es un aspecto clave en la medida que las empresas del

1 Di Benedetta, P., Lieberman, I., y L. Ard (2015). Corporate Governance in microfinance institutions. Word Bank. Washington

2 El Comité de Supervisión Bancaria en su documento Principios de gobierno corporativo para bancos (2015) señala que: "Un buen gobierno corporativo es esencial para el correcto funcionamiento del sector bancario y de la economia en su conjunto. Los bancos desempeñan un papel crucial en la economia mediante la intermediación de capital entre la economia mediante la intermediación de capital entre desarrollo empresarial y crecimiento económico (..) Deficiencias en el gobierno de los bancos con una influencia significativa en el sistema financiero pueden provocar la transmisión de problemas al sector bancario y a la economia en su conjunto".

3 Claessens, S. y B. Yurtoglu (2012). Corporate Governance and Development: An Update. Focus $N^{\circ} 10$. Global Corporate Governance Forum and IFC. Washington DC. sector financiero son importantes proveedores de recursos para toda la economía.

Dentro del sector financiero, los aspectos de gobierno corporativo también son objeto de un interés creciente. Di Benedetta y otros (2015) destacan cómo a medida que se incrementa la participación de las empresas del sistema financiero en la economía, los temas de gestión de carteras de productos, fuentes de financiamiento, competencia en diferentes mercados y estándares de gobierno corporativo se han vuelto más complejos. En muchos casos, los Estados han establecido requerimientos regulatorios que demandan mayor atención y capacidades de los directorios y las gerencias de las empresas del sistema financiero; en el caso del Perú, la experiencia de dichas empresas ha venido cobrando una importancia creciente y se requieren también fortalecer los temas de gobierno corporativo ${ }^{4}$.

Mehran, Morrison y Shapiro (2011) señalan que, de una recopilación de documentos que relacionan gobierno corporativo y riesgo, el tamaño del directorio tiene una relación ambigua con el riesgo. No obstante, ninguna variable mencionada sobre riesgo se relaciona con la morosidad y calidad de cartera como lo hace el presente documento.

\section{CARACTERÍSTICASDELDIRECTORIO E INDICADORES DE MOROSIDAD Y CALIDAD DE CARTERA. ALGUNAS HIPÓTESIS.}

El buen gobierno corporativo provee incentivos al Directorio y la Gerencia para establecer objetivos para las instituciones financieras que minimicen los conflictos de interés entre accionistas, directorio, gerencia, y demás grupos de interés. En este sentido, el Comité de Basilea (2015) estableció Principios sobre Gobierno Corporativo, entre ellos buenas prácticas para la actuación del directorio ${ }^{5}$.

De esta forma, en el presente documento se pretende responder la siguiente pregunta: ¿qué

\footnotetext{
4 Recientemente la Superintendencia de Banca, Seguros y AFP, mediante Resolución SBS 272-2017 del 18.01.2017, aprobó el Reglamento de Gobierno Corporativo y de la Gestión Integral Reglamento de Gobierno Corporativo y de la Gestion Integral
de Riesgos, en el que establece criterios referidos al director independiente, comités del directorio, sistema de remuneración, y la gestión de conflictos de intereses, entre otros. Los nuevos requerimientos regulatorios recogen determinados elementos de los Principios para el Fortalecimiento del Gobierno Corporativo (Principles for Enhancing Corporate Governance), que aprobó el Comité de Basilea y actualizó en julio de 2015.

5 Ver también Hagendorff, Jens (2015). Corporate governance in Banking. The Oxford Handbook of Banking
} 
características tienen los directorios y cómo éstos están asociados a indicadores de riesgo crediticio en una institución financiera? ${ }^{6}$.

Nuestro planteamiento es que, el nivel de riesgo de crédito de las instituciones financieras tiene una relación directa con las características de su gobierno corporativo, en particular con las características del directorio de la institución financiera.

De esta forma las hipótesis de trabajo son las siguientes: a) El número de directores que integran el directorio tiene una relación inversa con los ratios de morosidad y cartera pesada del portafolio de créditos de la institución financiera ${ }^{7}$. b) Asimismo, la presencia de mujeres en el directorio como proporción del total de miembros en el directorio tiene una relación inversa con la calidad y morosidad de la cartera de créditos; $y, c)$ El grado de asistencia de los directores al directorio permite que las decisiones que se toman tengan una mayor deliberación, y por lo tanto la toma de riesgos de considere con mayor cautela; se espera que, ante indicadores de mayor asistencia, los indicadores de morosidad y calidad de cartera de créditos sean menores.

\section{DATA Y METODOLOGÍA}

En el 2002 en el Perú, la Comisión Nacional Supervisores de Empresas y Valores (CONASEV), hoy Superintendencia del Mercado de Valores (SMV), publicó el documento "Principios de Buen Gobierno Corporativo para las Sociedades Peruanas", y posteriormente, en el 2005 aprobó las Normas Comunes para la Determinación del Contenido de los Documentos Informativos, aprobadas mediante Resolución Gerencia General N $N^{\circ}$ 211-98-EF/94.11 con el objetivo de solicitar a los emisores de valores inscritos en el Registro Público del Mercado de Valores información sobre el cumplimiento y grado de implementación de los "Principios de Buen Gobierno Corporativo para las Sociedades Peruanas".

En junio de 2014, la SMV reemplazó la "Información sobre el cumplimiento de los Principios de Buen Gobierno Corporativo

\footnotetext{
6 Ver Mersland, R. y R. Oystein (2009). Performance and Governance in Microfinance Institucions. Journal of Banking and Finance. 33 (4). También Gohar, R. y A. Batool (2015). Effect of Corporate Governance on Performance of Microfinance Institutions: A Case from Pakistan. Emerging Markets Finance \& Trade. $\mathrm{N}^{\circ} 51$

7 Gohar y Batool (2015) analizan variables como tamaño del directorio y presencia de mujeres en el directorio con el ROA.
}

para las Sociedades Peruanas" por el "Reporte sobre el Cumplimiento del Código de Buen Gobierno Corporativo para las Sociedades Peruanas", reporte que, como el anterior, es de carácter voluntario y contiene información revelada por las propias empresas respecto de los pilares que forman parte del cuestionario sobre el cumplimiento de las buenas Prácticas de gobierno corporativo.

En el presente documento, la información sobre el directorio (Pilar III) será analizada y asociada a indicadores cuantitativos de riesgo. El análisis sólo se realiza sobre el grupo de empresas que pertenecen al sistema financiero.

En tal sentido, la información de carácter público reportada en el cuestionario por las empresas del sistema financiero en el portal de la SMV como el número de directores, presencia de mujeres, y porcentaje de asistencia al directorio será analizada y relacionada a indicadores de morosidad y calidad de la cartera de créditos.

Con la finalidad de explicar el efecto de las prácticas de buen gobierno corporativo sobre el nivel de riesgo que enfrentan las empresas del sistema financiero peruano, se plantea el uso de variables cualitativas y cuantitativas mediante datos de panel y el uso de correlaciones.

La muestra utilizada está compuesta por las empresas del sistema financiero peruano que han remitido el Reporte de Información Sobre el Cumplimiento de Buen Gobierno Corporativo para las Sociedades Peruanas a la Superintendencia de Mercado de Valores (SMV). Dicho reporte, de periodicidad anual, ha sido declarado por las entidades con valores listados en la Bolsa de Valores de Lima (BVL). Dado que no todas las empresas del sistema financiero peruano han enviado este reporte, sólo se incluye la información disponible en la Bolsa de Valores de Lima (BVL) durante los años 2013 - 2017, de las siguientes empresas: 
Tabla 1

Relación de empresas con valores listados que publican el cuestionario

\begin{tabular}{lll}
\hline \hline & Banca múltiple & \multicolumn{1}{c}{ Financieras } \\
\hline \hline B. Azteca & Interbank & Compartamos Financiera \\
B. Cencosud & Ripley & Crediscotia Financiera \\
B. Comercio & B. Santander del Perú & Financiera Confianza \\
B. Crédito del Perú & B. Continental & Financiera Efectiva \\
B. Falabella del Perú & Citibank & Financiera Oh! \\
B. Financiero & Mi Banco & Financiera Proempresa \\
B. GNB & Scotiabank Perú & Financiera Qapaq \\
B. Interamericano de Finanzas & & Mitsui Auto Finance \\
\hline
\end{tabular}

Fuente: Elaboración Propia (se excluyen entidades estatales Cofide y BN)

Lainformaciónutilizada en el presentedocumento proviene de los reportes de Información Sobre el Cumplimiento de Buen Gobierno Corporativo para las Sociedades Peruanas, emitidas por las empresas con valores listados en la Bolsa de Valores de Lima; este reporte de autoevaluación de cada empresa se basa en El Código de Buen Gobierno Corporativo.

Las variables relevantes analizadas son las siguientes:

\section{Variables de gobierno corporativo.}

1. Tamaño de directorio: número total de miembros, incluyendo a los directores independientes.

2. Porcentaje de asistencia a las sesiones del directorio: Asistencia promedio de los directores a las sesiones del directorio.

\section{Variables de performance de riesgo crediticio.}

1. Calidad de la cartera de créditos (Ratio de cartera pesada): Indica los créditos otorgados por las empresas del sistema financiero con calificaciones de riesgo crediticio de deficiente, dudoso y pérdida. La información corresponderá a promedios anuales y para el periodo de análisis.

2. Morosidad de la cartera de créditos (Ratio de morosidad): Indica los créditos otorgados por las empresas del sistema financiero registradas en el balance de la institución financiera como vencidos y en cobranza judicial con relación al total de las colocaciones brutas. La información corresponderá a promedios anuales y para el periodo de análisis.

\section{Variables macroeconómicas.}

1. Inflación: Datos de inflación promedio anual tomado como índices de fin de periodo.

2. LNPBI: Logaritmo del PBI nominal expresado en millones de soles.

\section{Variables de control.}

1. Endeudamiento: Ratio de apalancamiento de cada empresa (pasivos/patrimonio) como promedio anual y para el periodo de análisis.

2. LNAT: Logaritmo de los activos totales de cada empresa como promedio anual y para el periodo de análisis.

Con la información pública de las instituciones financieras que reportan a la Superintendencia del Mercado de Valores, y con una base de datos que permite realizar un análisis de panel data, se han realizado correlaciones entre las variables de gobierno corporativo elegidas y los indicadores de morosidad y calidad de la cartera de créditos de la institución financiera.

\section{RESULTADOS}

El resultado de todas las correlaciones realizadas es que la calidad y la morosidad de la cartera de créditos están asociadas al tamaño del directorio, situación que indicaría que mientras más debate e intercambio de ideas diversas en el directorio, las decisiones sobre el riesgo crediticio asumido se evalúen mejor.

Correlación negativa entre la calidad (cartera pesada) y la morosidad de la cartera de créditos con el tamaño del directorio

Se observa una correlación negativa del 19.92\% entre la cartera pesada y el tamaño del directorio. 
Esto significa que, ante un mayor número de miembros en el directorio, es menor la cartera pesada que tiene la institución financiera, en parte, debido a que mientras más miembros sean parte del directorio, las decisiones tendrían una mayor deliberación que permita evaluar los riesgos crediticios y mitigarlos.

Asimismo, se observa una correlación negativa del $10.44 \%$ entre la morosidad y el tamaño del directorio. Esto implica que, ante un mayor número de miembros en el directorio, la morosidad con respecto a los créditos otorgados disminuye. Al igual que la correlación anterior, el tamaño de directorio afecta de la misma manera a la morosidad.

De esta forma, sí parece relevante que el número de directores que conforman el directorio tiene correlación negativa con los riesgos crediticios asumidos por las instituciones financieras, siendo más clara la correlación negativa entre tamaño del directorio y la cartera pesada.

\section{Correlación entre la proporción de mujeres en el directorio con indicadores de calidad (cartera pesada) y morosidad de la cartera de créditos}

Las correlaciones obtenidas no evidencian una clara asociación entre las mujeres en el directorio e indicadores de morosidad y calidad de la cartera de créditos. Se observa una correlación positiva del $6.26 \%$ entre la proporción de mujeres en el directorio y la morosidad. Ante un mayor número de mujeres en el directorio aumenta el ratio de morosidad de la empresa, situación que no parece alineada con otros trabajos que señalan que la participación de la mujer en el directorio sí permite una mejor gestión del riesgo en la institución financiera.

Asimismo, al asociar la variable proporción de mujeres y la calidad de la cartera, se observa una correlación positiva del $0.47 \%$ entre la proporción de mujeres en el directorio y la cartera pesada; de esta forma, siendo la correlación cercana a cero se podría esperar que estas dos variables tengan una correlación muy poco significativa.

De esta forma, se aprecia una muy baja asociación entre las variables analizadas, y particularmente entre la participación de las mujeres y la cartera pesada; por lo que, con la información analizada, no existe una clara correlación o se evidencia correlación no significativa entre la participación de las mujeres en el directorio y los indicadores de morosidad y calidad de la cartera de créditos de una institución financiera.

Correlación entre la cartera pesada y morosidad con el ratio de asistencia de los miembros a los directorios

Se observa una correlación positiva del $2.38 \%$ entre la cartera pesada y el número de asistencia de los directores a las reuniones de directorio, y de una correlación positiva del $1.02 \%$ entre el número de asistencia de los directores a las reuniones de directorio y la morosidad. Ambas correlaciones son muy bajas, por lo que, con la información analizada, no es clara la correlación entre las variables analizadas.

Tabla 2

Matriz de correlaciones

\begin{tabular}{|c|c|c|c|c|c|c|c|c|c|}
\hline & $\begin{array}{c}\text { CARTERA } \\
\text { PESADA }\end{array}$ & ASISTENCIA & $\begin{array}{c}\text { BOARD_ }_{-} \\
\text {SIZE }\end{array}$ & ENDEUDAMIENTO & INFLACION & LNAT & LNPBI & MOROSIDAD & MUJERES \\
\hline $\begin{array}{c}\text { CARTERA } \\
\text { PESADA }\end{array}$ & 1.000 & & & & & & & & \\
\hline ASISTENCIA & $\begin{array}{c}0.024 \\
{[0.503]}\end{array}$ & 1.000 & & & & & & & \\
\hline BOARD_SIZE & $\begin{array}{c}-0.199 * * * \\
{[-2.463]}\end{array}$ & $\begin{array}{c}0.044 \\
{[-0.977]}\end{array}$ & 1.000 & & & & & & \\
\hline ENDEUDAMIENTO & $\begin{array}{c}-0.500^{* * *} \\
{[-8.983]}\end{array}$ & $\begin{array}{c}-0.046 \\
{[-0.518]}\end{array}$ & $\begin{array}{c}0.2304 * * * \\
{[2.852]}\end{array}$ & 1.000 & & & & & \\
\hline INFLACION & $\begin{array}{c}-0.006 \\
{[-0.046]}\end{array}$ & $\begin{array}{c}0.025 \\
{[-0.942]}\end{array}$ & $\begin{array}{c}0.060 \\
{[0.597]}\end{array}$ & $\begin{array}{c}0.089 \\
{[0.236]}\end{array}$ & 1.000 & & & & \\
\hline LNAT & $\begin{array}{c}-0.609 * * * \\
{[-10.959]}\end{array}$ & $\begin{array}{c}0.100 \\
{[0.044]}\end{array}$ & $\begin{array}{l}0.295^{* *} \\
{[2.699]}\end{array}$ & $\begin{array}{l}0.719 * * * \\
{[12.921]}\end{array}$ & $\begin{array}{c}-0.024 \\
{[-0.464]}\end{array}$ & 1.000 & & & \\
\hline
\end{tabular}




\begin{tabular}{|c|c|c|c|c|c|c|c|c|c|}
\hline \multirow{2}{*}{ LNPBI } & 0.075 & -0.154 & -0.160 & $-0.219^{* *}$ & $-0.513^{* * *}$ & 0.006 & 1.000 & & \\
& {$[1.521]$} & {$[-1.419]$} & {$[-1.307]$} & {$[-2.306]$} & {$[-3.084]$} & {$[0.214]$} & & & \\
\hline \multirow{2}{*}{ MOROSIDAD } & $0.896 * * *$ & 0.010 & -0.104 & $-0.496^{* * *}$ & -0.010 & $-0.577^{* * *}$ & 0.117 & 1.000 & \\
& {$[24.970]$} & {$[0.236]$} & {$[-1.557]$} & {$[-7.312]$} & {$[0.431]$} & {$[-8.653]$} & {$[1.519]$} & & \\
\hline \multirow{2}{*}{ MUJERES } & 0.005 & 0.208 & 0.133 & 0.017 & -0.128 & 0.014 & 0.217 & 0.063 & 1.000 \\
& {$[0.0154]$} & {$[0.230]$} & {$[0.216]$} & {$[0.103]$} & {$[-0.037]$} & {$[0.089]$} & {$[0.158]$} & {$[0.126]$} & \\
\hline
\end{tabular}

* Significativo al $10 \%$

** Significativo al 5\%

*** Significativo al $1 \%$

[ ] = Spearmen $\mathrm{t}$-stat

Fuente: Elaboración Propia

\section{CONCLUSIONES}

El principal resultado es que el tamaño del directorio tiene una correlación negativa con los indicadores de calidad y morosidad de la cartera de créditos. Un mayor número de miembros de un directorio permite una deliberación e intercambio de ideas que permitiría mayor prudencia en la toma de riesgos, por lo que los indicadores de calidad y morosidad de la cartera de créditos son menores.

Sin embargo, otras variables de gobierno corporativo como porcentaje de mujeres en el directorio y el porcentaje de asistencia de los directores a las sesiones del directorio no parecen ser variables que tienen alguna correlación con los indicadores de cartera, al menos en bancos $\mathrm{y}$ financieras, que fueron las instituciones analizadas.

Si bien las variables tamaño del directorio e indicadores de cartera están correlacionadas, se esperaría que una mayor asistencia a las sesiones del directorio esté correlacionada también a la cartera, no obstante la correlación es no significativa. Quizás en este caso habría que validar la información reportada sobre asistencia al directorio.

En el análisis de bancos y financieras, la variable mujeres en el directorio no ha tenido alguna significancia, como también lo señalan Gohar y Batool (2015) en su trabajo sobre el efecto del gobierno corporativo en la instituciones microfinancieras en Pakistán, en el cual se indica que la presencia de mujeres en el directorio no tiene mayor importancia en la mejora de la performance económica. Por otro lado, de acuerdo a Mersland y Strom (2009), sí existe una relación significativa y positiva entre una $\mathrm{CEO}$ mujer y el ROA. A pesar de ello, se recomienda analizar esta información en las instituciones microfinancieras.
Debemos señalar que, las instituciones analizadas sólo han sido bancos y financieras debido a que sólo ellas remiten información periódica a la Superintendencia del Mercado de Valores (SMV) a través del Cuestionario. No se tiene información pública en el portal de la SMV sobre las cajas municipales, cajas rurales y Edpymes, por lo que con este tipo de información se podría profundizar el análisis.

Un aspecto que hay que destacar es que el análisis se ha realizado sobre la información que las propias instituciones financieras remiten a la SMV, de esta forma, se ha asumido que la información es la correcta; sin embargo, esta información debería ser validada por terceros.

\section{REFERENCIAS}

[1] Abdulazeez, DA*, Ndibel y Mercy, AM. (2016). Corporate Governance and Financial Performance of Listed Deposit Money Banks in Nigeria. Journal of Accounting \& Marketing. 5(1). doi: 10.4172/21689601.1000153

[2] Berg, T. (2015). Playing the devil's advocate: The causal effect of risk management on loan quality. The Review of Financial Studies, 28(12), pp. 3367-3406. doi: https:// doi.org/10.1093/rfs/hhv040

[3] Cheung, Y., Connelly, J., Limpaphayom, P. y Zhou, L. (2005). Do investors really value Corporate Governance. Evidence from the Hong Kong Market. Hong Kong Institute for Monetary Research, (222005).

[4] Claessens, S. y Yurtoglu, B. (2012). Corporate governance and development: an update. Washington DC: Focus 10, Global Corporate Governance Forum and The International Finance Corporation. 
[5] De Haan, J., y Vlahu, R. (2013). Corporate Governance of Banks: A survey. De Nederlandsche Bank, (386).

[6] Deloitte. (2016). Good governance driving corporate performance? A meta-analysis of academic research \& invitation to engage in the dialogue. Recuperado de https://www2.deloitte. com/content/dam/Deloitte/nl/Documents/risk/ deloitte-nl-risk-good-governance-drivingcorporate-performance.pdf

[7] Di Benedetta, P., Lieberman, I., y Ard, L. (2015). Corporate governance in microfinance institutions. Word Bank Group, (96022).

[8] Gohar, R. y Batool, A. (2015). Effect of corporate governance on performance of microfinance institutions: A case from Pakistan. Journal Emerging Markets Finance \& Trade, 51. doi: https://doi.org/10.1080/154 0496X.2015.1080559

[9] Harm, C. (2002). Bank management between shareholders and regulators. Vienna, Austria: Société Universitaire Européenne de Recherches Financiéres.

[10] Hagendorff, Jens (2015). Corporate governance in banking. En The Oxford Handbook of Banking, Edited by Allen N. Berger, Phillip Molyneux, and John O.S. Wilson (Second Edition).

[11] Lefort, F. (2003). Gobierno Corporativo: Qué es? y Cómo andamos por casa? Cuadernos de Economía, 40(120), pp. 207237. doi: http://dx.doi.org/10.4067/S071768212003012000002

[12] Maher, M. y Andersson, T. (1999). Corporate governance: effects on firm performance and economic growth. Organization for Economic Co-Operation and Development (OECD). Recuperado de: https://www.oecd.org/sti/ind/2090569.pdf

[13] Mehran, H. y Mollineaux, L. (2012). Corporate Governance of Financial Institutions. Federal Reserve Bank of New York, 539.
[14] Mehran, H., Morrison, A., y Shapiro, Joel. (2011). Corporate Governance and Banks: What Have We Learned from the Financial Crisis? Federal Reserve Bank of New York, 502 .

[15] Mehran, H., y L. Mollineaux (2012). Corporate Governance of Financial Institution. Federal Reserve Bank of New York, 539.

[16]Mersland, R. y Oystein, R. (2009). Performance and Governance in Microfinance Institutions. Journal of Banking and Finance, 33 (4).

[17] Minton, B., Taillard, J. y Williamson, R. (2014). Financial Expertise of the Board, Risk Taking, and Performance: Evidence from Bank Holding Companies. Journal of Financial and Quantitative Analysis. 49(2), pp. 351-380.

[18] Mülbert, P. (2010). Corporate governance of banks after the financial crisis - Theory, Evidence, Reforms. ECGI Working Paper, 151.

[19] Superintendencia de Mercado de Valores. (2013). Código de Buen Gobierno Corporativo para las Sociedades Peruanas. Recuperado de: http://www.smv.gob.pe/ Uploads/CodBGC2013\%20_2_.pdf

[20] Stenning, F., Boza, B. (2015). Buenas prácticas de Gobierno Corporativo. La Voz del Mercado.

[21] Stulz, R. Z. (2016). Risk Management, Governance, Culture, and Risk Taking in Banks. Recuperado de : https://pdfs. semanticscholar.org/1a5c/3dc62208f615b7 c171fdb9656ca57f006139.pdf

[22] TShipa, J. (2017). Corporate Governance and Financial Performance: A study of companies listed on the Johannesburg Stock. University of Pretoria. 